Aliaga, F.M.; Gutiérrez-Braojos, C. y Fernández-Cano, A. (2018). Las revistas de investigación en educación: Análisis DAFO. Revista de Investigación Educativa, 36(2), 563-579.

DOI: http://dx.doi.org/10.6018/rie.36.2.312461

\title{
Las revistas de investigación en educación: Análisis DAFO
}

\section{Research journals in education: SWOT Analysis}

\author{
Francisco M. Aliaga*, Calixto Gutiérrez-Braojos*, Antonio Fernández-Cano* \\ * Universidad de Valencia, Departamento de Métodos de Investigación y Diagnóstico en Educación \\ ** Universidad de Granada, Departamento de Métodos de Investigación y Diagnóstico en Educación
}

\section{Resumen}

Introducción: Se revisa la evolución, situación actual y perspectivas de las revistas de investigación sobre educación, haciendo énfasis sobre las publicadas en español. Metodología: Se utiliza la estructura de la metodología DAFO (debilidades, amenazas, fortalezas y oportunidades), que ha demostrado ser una técnica muy eficaz para analizar y formular estrategias de acción que conduzcan a la mejora de un producto o servicio. Resultados: Se identifican las principales debilidades, amenazas, fortalezas y oportunidades de las revistas de investigación en educación, señalando los elementos fundamentales, internos y externos, que influyen sobre su desarrollo y calidad. Discusión: Se presentan conclusiones sobre el futuro de las revistas de investigación educativa como instrumento de comunicación científica, señalando opciones de mejora y propuestas para aprovechar mejor este potente recurso de comunicación y difusión del conocimiento científico. El ámbito de las revistas iberoamericanas se revela como un campo dinámico y complejo, con múltiple problemas pero también con retos y grandes posibilidades.

Palabras clave: Publicaciones; Revistas; Evaluación; Publicaciones Científicas; Publicaciones Electrónicas.

Correspondencia: Francisco M. Aliaga, Universidad de Valencia, Departamento de Métodos de Investigación y Diagnóstico en Educación, Facultad de Filosofía y Ciencias de la Educación, Av. Blasco Ibáñez, 30, 46010, Valencia. Correo electrónico: franscisco.aliaga@uv.es 


\begin{abstract}
Introduction: This review analyze the evolution, the present-day situation and the perspectives of research journals on education, putting emphasis on those published in Spanish. Methodology: The structure of the SWOT methodology (Strengths, Weaknesses, Opportunities and Threats) is used, which has proven to be a very effective technique to analyze and formulate implementation strategies that arrive at the best possible outcomes for a given product or service. Results: We identify the principle weaknesses, threats, strengths and opportunities of the education research journals, pointing to the fundamental elements, both internal and external, that influence their development and quality. Discussion: We will draw conclusions about the future of the educational research journals as an instrument of scientific communication, as future improvements/advancements and proposals to better utilize this potent resource of communication and scientific knowledge are made. The scope of Ibero-American journals show that it is a dynamic and complex field, with assorted problems but also with challenges and great possibilities.

Keywords: Publications; Journals; Evaluation; Scholastic Journalism; Electronic Journals.

Las publicaciones periódicas tienen una larga tradición en el ámbito científico, que arranca con Journal des Savants y Philosophical Transactions of the Royal Society en 1665. Han acabado imponiéndose, en años recientes, tanto en respuesta a los procesos de evaluación institucional (basados prioritariamente en análisis de citas de revistas), como debido a las características editoriales de las revistas, que presentan un buen equilibrio entre rapidez de edición, amplitud de contenido y accesibilidad (frente al libro, mucho más lento y menos accesible, o a las comunicaciones a Congresos, más rápidas pero con limitaciones de contenido y problemas de acceso).

Son varias las funciones que cubren las revistas. Por un lado permiten difundir la ciencia, distribuyendo el nuevo conocimiento entre la comunidad científica y, por otro lado, divulgar la ciencia, haciendo que llegue ese conocimiento al conjunto de la sociedad (Cabrera-Flores, Luna-Serrano \& Vidauri, 2014). Desde otra perspectiva, Martínez-Rizo (1999) planteó una tipología de las revistas que diferencia entre a) boletines o gacetas (centrados en la difusión inmediata de información a públicos especializados), b) revistas de divulgación (centradas en la comprensibilidad por parte de legos) y c) revistas académicas, que publican resultados de investigación y poseen filtros de calidad (arbitradas). En el presente artículo nos centramos exclusivamente en el análisis de las revistas de investigación.

El propósito de este trabajo es presentar un análisis sobre las revistas de investigación educativa, fundamentalmente desde una perspectiva iberoamericana, aplicando la estructura DAFO tradicional (debilidades, amenazas, fortalezas y oportunidades).

El DAFO es un método analítico que surgió en el ámbito empresarial en los años 60, y que se ha extendido rápidamente a otras áreas (Hill \& Westbrook, 1997). Pretende analizar los principales factores que implican un producto, servicio u organización, con el fin de formular estrategias de acción para su mejora (Chang \& Huang, 2006; Lee \& Lin, 2008). Por tanto, es una herramienta propia del pensamiento estratégico, válida para el diagnóstico y la planificación, y no un instrumento meramente descriptivo.
\end{abstract}


Este método plantea que una manera eficiente de construir estrategias de mejora consiste en el análisis confrontado de factores internos y externos. El DAFO supone representar de manera organizada cada factor en una matriz $2 \times 2$ (Tabla 1). Los factores internos pueden ser atributos positivos (fortalezas) o negativos (debilidades). El análisis de los factores externos analiza elementos independientes al objeto valorado, pero que podrían influir positivamente (oportunidades) o negativamente (amenazas) sobre él, como por ejemplo, aspectos políticos, económicos o tecnológicos (Wang, Zhang \& Yang, 2014).

Tabla 1

Matriz DAFO

\begin{tabular}{lcc}
\hline & Valor positivo & Valor negativo \\
\hline Análisis interno & Fortalezas & Debilidades \\
Análisis externo & Oportunidades & Amenazas \\
\hline
\end{tabular}

En este artículo hemos optado por un análisis DAFO de estilo narrativo, que nos permita razonar más detalladamente y presentar esas reflexiones para el debate.

La distinción entre interno y externo puede tener límites ambiguos y difusos en el caso de las revistas científicas, ya que no son un ente autónomo, sino que forman parte de un sistema global más amplio, que incluye el proceso editorial (gestión, autoría, revisión, etc.), pero también a las instituciones de las que forman parte y al propio sistema de investigación. Delimitar qué es interno y qué es externo, cuando las influencias y procesos están en continua interacción, puede resolverse de maneras diversas según el enfoque.

\section{Fortalezas}

Como hemos comentado, existe consenso en reconocer a las revistas científicas como un instrumento adecuado para lograr el mayor impacto de los hallazgos científicos de los investigadores educativos. Este impacto comprende tanto a la propia comunidad científica como a las políticas educativas, que deberían basarse en esas evidencias para tomar decisiones (Mañana \& Giménez, 2015).

Sin descartar otras, cualquier investigador dispone en la actualidad de una amplia variedad de revistas educativas iberoamericanas a las que enviar sus trabajos para publicar. De hecho, el sistema de información sobre revistas científicas Lantindex incluye un total de 988 revistas educativas, bastante concentradas en determinados países (véase Figura 1).

Varios son los factores internos que hemos identificado y que explican esta proliferación de revistas educativas. Uno de ellos es que la mayoría de las revistas científicas han incrementado la difusión de su contenido en formato digital. Este formato ofrece un menor coste de distribución con respecto al impreso, unido a ventajas de procesamiento, distribución, difusión, almacenamiento y recuperación, por lo que se está 


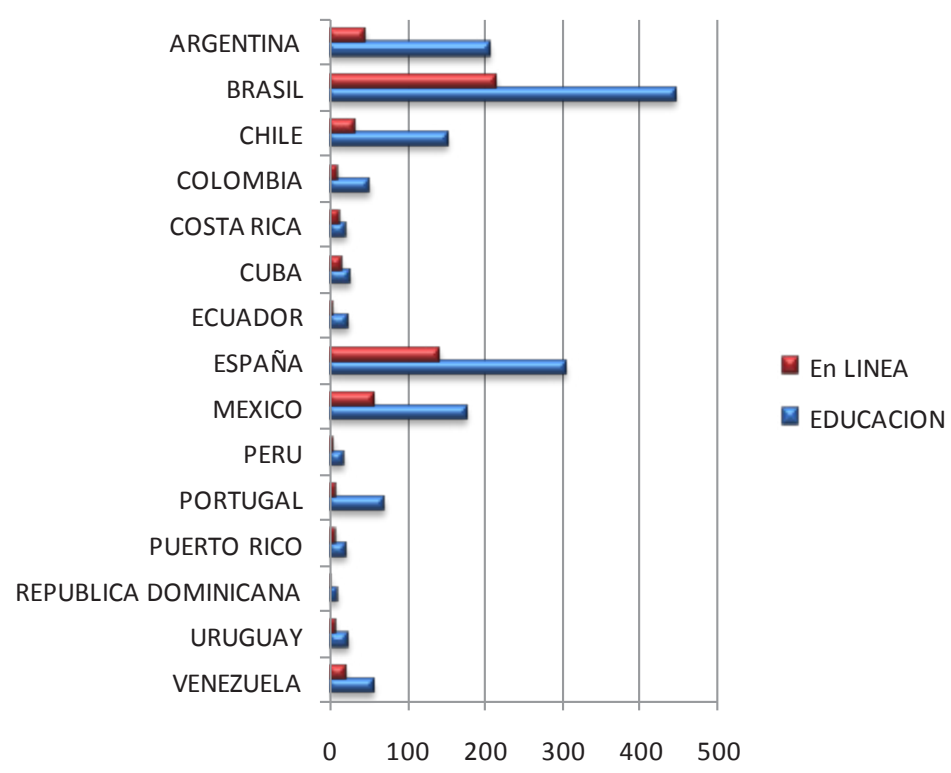

Figura 1. Número de revistas de educación y específicamente En Línea en Latindex por país.

Principales países productores (más de 10 revistas de educación)

consolidando como canal prioritario (Kling \& Callahan, 2003). De hecho, actualmente están contabilizadas en Latindex un total de 329 revistas educativas en formato digital, y otras siguen haciendo el trasvase de formato.

El formato digital permite incluir contenido multimedia (texto, imagen, audio y video) en las producciones. Entre otras ventajas, supone una difusión y acceso más ágiles, ya que en cuanto se genera el contenido digital, es accesible desde cualquier parte por muchos usuarios, sincrónicamente, gracias a internet. Ello permite que revistas educativas que tradicionalmente imprimían tiradas muy cortas y tenían problemas de distribución (Díaz Barriga, 2000) alcancen ahora, a menor coste, un público mucho mayor.

Como ventaja adicional, las instituciones prefieren adquirir producción científica en formato digital, ya que con ella se reduce considerablemente el espacio físico de almacenamiento y se facilita la actividad de búsqueda, mediante procesos integrados de búsqueda y recuperación de documentos.

Las revistas científicas carecen de capacidad para procesar y publicar la totalidad de manuscritos recibidos. Tampoco sería deseable, pues suponen un filtro competitivo a las producciones, y un mecanismo para difundir solo los artículos de mayor calidad y eliminar aquellos que incumplen con la ética profesional (p. ej. plagios, ataques ad hominem, etc.).

Un fenómeno innegable es la mejora progresiva de la calidad de las revistas debido a diversas iniciativas de evaluación externa. América Latina fue pionera en procesos regionales de evaluación y reconocimiento de calidad de revistas científicas, con iniciativas como Latindex o Scielo, que mantienen e incluso incrementan el reconocimiento institucional y social en los últimos tiempos (integración de Scielo en Web 
of Science). También en este lado del Atlántico han sido muchas las iniciativas, tanto a nivel europeo -ERIH- (Aliaga, Almerich \& Suárez-Rodríguez, 2013) como español -In-RECS, In-RECH, DICE, RESH - (Delgado López-Cózar, 2015; Ruiz-Corbella, Galán \& Diestro, 2014). Estos sistemas de evaluación contaron con financiación oficial y con el reconocimiento institucional que acreditaba su calidad, de modo que han ayudado a los editores a mejorar sus publicaciones y a los autores a elegir revistas.

Observamos, en resumen, un aumento cuantitativo y cualitativo de las revistas académicas de educación disponibles, a la vez que se ha desarrollado la accesibilidad, difusión y manejabilidad de la información publicada.

\section{Debilidades}

A diferencia de otras disciplinas científicas con robustas fuentes de financiación externas (farmacología, medicina, química, física, etc.), no existe un mercado comercial que sustente el campo de las revistas en el ámbito de la mayoría de las Ciencias Sociales (Delgado, 2014). En un estudio realizado sobre revistas españolas de Humanidades y Ciencias Sociales, Rodríguez-Yunta y Giménez-Toledo (2013) encontraron que un $38,1 \%$ de las mismas son editadas por universidades públicas. Si a ello añadimos las revistas promovidas y financiadas directamente por la Administración (ministerios, etc.), alcanzamos un 58,5\%, es decir, una holgada mayoría del panorama existente. También en Latinoamérica el panorama viene dominado por instituciones públicas (Delgado, 2011, 2014; Fischman, Alperín \& Willinsky, 2010). En época de crisis económica (o por cambio de las políticas) la financiación de estas revistas puede verse seriamente comprometida, amenazando la supervivencia de muchas de ellas. Ello demuestra la debilidad económica estructural de estas publicaciones, lo que explica en buena medida su alta tasa de mortalidad. Por tanto, este factor económico no debe considerarse como 'externo', sino que determina su nacimiento, sus probabilidades de pervivencia y, frecuentemente, su extinción.

En este contexto económico, la creación (y mantenimiento) de revistas científicas de educación frecuentemente ha venido siendo una tarea ardua, a veces casi milagrosa (Delgado López-Cózar, 2015). Con frecuencia, la gestión de una revista se convierte; en una tarea adicional (y, normalmente, no remunerada - Delgado, 2014) al trabajo habitual (docencia y/o investigación). Ello conlleva dos debilidades principales. Por un lado una escasa formación inicial de los gestores de las revistas (la actividad editorial es muy diferente a la docente o investigadora en la que se han formado). Sólo grandes dosis de ilusión (y de coste personal, en forma de tiempo y sinsabores) permiten suplir las carencias en ese campo. El segundo problema tiene que ver con la fuerte dependencia de estas aventuras editoriales con alguna persona concreta. Este personalismo supone un gran riesgo para la pervivencia de las revistas cuando, por algún motivo (agotamiento, jubilaciones, etc.), su responsable abandona la gestión. Esto se convierte en un problema grave, debido a que cuanto más crece una revista (en prestigio, en reconocimiento, en indexación) mayor se hace la carga de trabajo (Delgado, 2014), aunque con incentivos prácticamente inexistentes (Delgado López-Cózar, 2015).

Las limitaciones económicas de las revistas científicas de educación (y, en realidad, de Ciencias Sociales) condicionan, indirectamente, una debilidad adicional de las 
mismas. Tratándose, en general, de iniciativas modestas, sin apenas infraestructura, lo que se suelen desarrollar en nuestro ámbito son proyectos editoriales aislados. Han de competir con enormes corporaciones comerciales que ofrecen "paquetes" de cientos de revistas (algo que a las bibliotecas universitarias les simplifica mucho la gestión), que incluyen sistemas integrados de valor añadido (buscadores, etc.). Si bien las revistas de investigación educativa son un aspecto casi marginal para esas empresas, las que forman parte de esos paquetes comerciales cuentan con la ventaja incomparable de una gestión profesional (Delgado, 2014) y tienen oportunidades de difusión que no pueden alcanzar los proyectos aislados de revistas científicas. Aunque la calidad de los contenidos de este último tipo de revistas sea excelente, su alcance y difusión siempre será mucho más débil, limitando tanto su impacto académico como social. Se trata de un enfrentamiento desigual que solo el esfuerzo en pos de la excelencia en la calidad editorial y la perseverancia permiten disputar con ciertas opciones de éxito. Para visualizar este desequilibrio de fuerzas (Fonseca-Mora \& Aguaded, 2014), baste decir que de 219 revistas incluidas en el Social Sciences Citations Index (SSCI), en la categoría de Education \& Educational Research, un total de 160 (el 72\% del total) están editadas por grandes corporaciones comerciales como Elsevier, Springer, Taylor \& Francis, o Sage (Ruiz-Corbella, Galán \& Diestro, 2014). Sin embargo, análisis recientes (Diestro, Ruiz Corbella \& Galán, 2017; Mañana \& Giménez-Toledo, 2015) revelan que el mayor coste de las revistas editadas por empresas con ánimo de lucro no aportan a los artículos que publican mayor valor añadido, en términos bibliográficos (precio por artículo, precio por cita, índice compuesto de precio e índice relativo de precio), respecto a las editadas por entidades sin ánimo de lucro, lo que pone en cuestión cuál es la aportación real de esas entidades comerciales y la justificación social de sus beneficios.

Merton (1973) planteó con claridad que la actividad de los científicos está orientada por su motivación hacia la construcción de conocimiento científico, el prestigio académico o social y las mejoras económicas y laborales. Seleccionar dónde publicar un artículo de investigación es una tarea compleja, que depende de una gran variedad de factores (Delgado Troncoso et al., 2014), pero básicamente predomina el factor de cómo conseguir el mayor reconocimiento posible, en base al prestigio de la revista (Fernández-Cano \& Bueno, 2002). En un entorno institucionalizado, el prestigio académico proviene en primer lugar del reconocimiento oficial del medio editorial en el que se publique, ya que permiten al investigador progresar en su carrera profesional o acceder con mayor facilidad a recursos para seguir investigando, mientras que al Centro en el que trabaja le posibilita mejoras en el prestigio.

La estabilidad en la valoración de este reconocimiento oficial posibilitaría a los investigadores tomar decisiones sobre el medio en el que publicar para maximizar sus esfuerzos. Sin embargo, tal estabilidad está lejos de conseguirse. Cambios políticos o, sencillamente de personal en los organismos oficiales dedicados a la evaluación de la investigación, suponen inestabilidad en los criterios. Así, por ejemplo, en España los criterios que se utilizan para evaluar a los investigadores son modificados, año a año, por el organismo encargado del proceso (CNEAI). No son necesariamente cambios radicales, pero puesto que se aplican a la evaluación de investigación realizada (y publicada) en años anteriores, sí se producen cambios que convierten en inútiles los esfuerzos realizados por muchos profesionales, dejándoles fuera de los nuevos estánda- 
res marcados a posteriori, fuera del control de sus protagonistas, generando indefensión aprendida (Seligman, 1975) e induciendo un comportamiento pasivo.

Queremos resaltar las contradicciones y la falta de apoyo institucional a diversas iniciativas de evaluación de la investigación, tanto en proyectos europeos (ERIH) como específicamente en el caso español (In-RECS, In-RECH, DICE, RESH). Al inicio de la presente década se produjo un cambio sustancial en las políticas que daban respaldo a estas iniciativas, lo que ha provocado su desmantelamiento por estrangulamiento económico (no achacable a la crisis económica, ya que se han seguido dedicando recursos incomparablemente mayores a otras iniciativas comerciales mucho más costosas: Aliaga, 2014; Giménez-Toledo, 2014). Este proceso ha sido especialmente perjudicial para el ámbito de las Ciencias Sociales y las Humanidades, al desaparecer prácticamente los medios de evaluación más contextualizados, algo absolutamente necesario en los ámbitos de investigación más ligados a una sociedad concreta, y por tanto no necesariamente objeto de interés más allá de sus fronteras. Por suerte, la tendencia es opuesta en Latinoamérica, donde Latindex o Scielo se consolidan.

Los cambios anteriormente mencionados en la política institucional suponen una debilidad añadida para la supervivencia de las revistas a largo plazo. La desaparición de los índices que habían venido acreditando la calidad y/o impacto de una revista suponen un desánimo en los responsables y una consiguiente falta de propuestas de artículos (en un entorno muy competitivo) que ponen en cuestión, incluso, su propia existencia.

Una de las consecuencias de esas decisiones de los policy makers ha sido que, en buena parte de las evaluaciones institucionales y personales de los investigadores, el grueso del peso del reconocimiento de la calidad e impacto de las revistas se ha dejado en manos de las dos principales empresas multinacionales que copan ese mercado: Clarivate Analytics (antes, Thomson-Reuters) a través de los Journal Citation Reports (JCR) y la editorial Elsevier, a través de Scopus. Se trata de unos indicadores de prestigio, con un enorme coste económico para los contribuyentes. Desde el punto de vista académico tienen un inconveniente adicional: se trata de instituciones privadas con ánimo de lucro, legítimo, pero alejado de los criterios científicos que deberían imperar en este campo. Así, por poner sólo un ejemplo, fue una decisión comercial (que no científica) la que indujo en 2007 a Thomson-Reuters de manera súbita (ante la creciente competencia de Scopus, menos anglocéntrica que su rival y más sensible a CC. SS.) a incrementar muy sustancialmente su cobertura de otros idiomas distintos del inglés, de otros países (hasta entonces virtualmente excluidos: ver Tabla 2) y de otros ámbitos científicos, lo que hizo crecer, por ejemplo, el número de revistas de educación incluidas en JCR en un 223\% (Aliaga, 2014; Purnell \& Quevedo-Blasco, 2013; Testa, 2011).

Una demostración simple, pero efectiva, de que esos incrementos de cobertura del JCR no pueden deberse a mejoras en la calidad editorial, en la investigación o en el impacto, sino a decisiones extra-científicas puede comprobarse en la Tabla 2. Hay que hacer notar que durante el periodo señalado España inició una exitosa política de negociación comercial, unificando las suscripciones de todas las universidades del país (a través de la FECYT, Fundación para la Ciencia y la Tecnología) y convertirse así en el segundo mayor cliente mundial de sus productos bibliométricos, lo que le permitió plantear una consideración más positiva de sus revistas nacionales. 


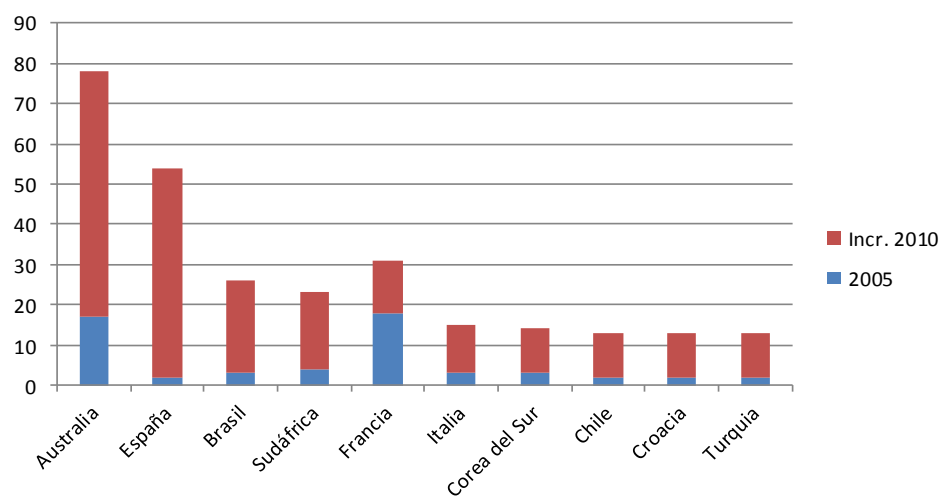

Figura 2. Crecimiento de revistas incluidas en JCR de algunos países en el periodo 2005-2010. Tomado de Testa (2011)

Tabla 2

Porcentaje de incremento de revistas incluidas en JCR de algunos países (2005-2010)

\begin{tabular}{lc}
\hline \multicolumn{1}{c}{ País } & Incremento de cobertura \\
\hline España & $2.600 \%$ \\
Brasil & $767 \%$ \\
Chile & $550 \%$ \\
Croacia & $550 \%$ \\
Turquía & $550 \%$ \\
Sudáfrica & $475 \%$ \\
Italia & $400 \%$ \\
Corea del Sur & $367 \%$ \\
Australia & $359 \%$ \\
Francia & $72 \%$ \\
\hline
\end{tabular}

Nota: Calculado a partir de los datos de Testa (2011)

Obviando estas influencias espurias, las bases de datos de Clarivate y Elsevier tienen otro problema adicional, se centran en la denominada "corriente principal" de la ciencia (Cabrera-Flores, Luna-Serrano \& Vidauri, 2014). Es, como mínimo, motivo de discusión si en el ámbito de las Ciencias Sociales existe, o debe existir, una "corriente principal" que nos lleve a todos hacia un mismo lugar. Aunque una parte del conocimiento en estas disciplinas es de interés general, e incluso común (como la metodología o la educación comparada), no es menos cierto que muchos subcampos tienen perfiles (intereses, condicionantes, contextos, resultados, etc.) propios de cada sociedad. Es una diferencia muy sustancial con respecto a las Ciencias Naturales, que sí comparten sus resultados. La aplicación de una ley educativa concreta a un país no tiene aplicación directa en otras culturas, en las que las condiciones materiales, los hábitos sociales, los objetivos o los participantes son diferentes. 
En el ámbito educativo tenemos entornos culturales propios y diferenciados con dinámicas distintivas. Debemos decidir si la investigación sobre cada uno de esos sistemas educativos debe estar enraizada y servir a la comunidad en la que se produce (por algo la denominamos ciencia Social: tiene sentido en la propia sociedad) o, por el contrario, debe formar parte de esa corriente principal unificada. Debemos decidir si esa peculiaridad propia de cada sociedad específica es una debilidad o una riqueza que debemos preservar.

Existen implicaciones prácticas evidentes de este dilema. Entre otros, el tema de si debemos usar el idioma vernáculo de cada comunidad para expresar los hallazgos de la investigación, para llegar más fácilmente a la sociedad en la que se incardina (Ishikawa, 2014). También afecta a si se van a considerar relevantes las revistas de enfoque más local o sólo aquellas que pretendan orientarse a un público internacional. Como vemos, se trata de temas complejos y no resueltos, excepto, quizás, como hechos consumados de los gestores de las evaluaciones y las políticas científicas, recibida con incomodidad por buena parte de la comunidad académica, lo que en ocasiones ha acabado en algo parecido a un plante, como el de Taiwan (Chou, 2014), en el que más de 3.000 investigadores de Ciencias Sociales se rebelaron contra la aplicación exclusiva del SSCI para evaluar su producción científica, consiguiendo cambios relevantes (aceptación de revistas locales de calidad reconocida y en idioma vernáculo). Críticas aún más antiguas a la aceptación unívoca de indicadores emitidos por las diversas bases del entonces Institute for Scientific Information (ISI) para evaluar la investigación educativa, sus revistas, agentes e instituciones, son localizables en Fernández-Cano (1995a, 1995b).

Una debilidad de las revistas de investigación (no sólo las educativas) que aún persiste es la resistencia institucional a los cambios, expresada de diversos modos. Por un lado, durante muchos años ha habido una oposición de los grupos más conservadores de la Academia (que suelen coincidir con los que están en la cúspide y que, como veteranos, se desenvolvieron en otras condiciones) a novedades relevantes como, por ejemplo, las revistas electrónicas. Como bien indica Delgado López-Cózar (2015), la especie predominante en esa época en la Academia era la del "homo tipographicus", que sólo valoraban a aquello que veían impreso. La desconfianza ante lo que se percibía como inmaterial, quizás efímero, supuso una penalización en los procesos de evaluación de la investigación para los artículos de revistas electrónicas (en España, por ejemplo, aún se penaliza en algunas disciplinas de la evaluación de los sexenios, requiriendo pruebas de calidad más exigentes que para las impresas). Esta resistencia ha provocado un desarrollo más débil en esos nuevos medios de comunicación.

Otra debilidad se refiere a la lentitud en el desarrollo y adopción de los formatos de edición, debido a las resistencias institucionales al cambio. El peso de una historia con textos impresos se hace sentir (Delgado López-Cózar, 2015), de modo que desde diversas instancias (editoriales universitarias, bases de datos, hemerotecas) se potencian formatos más rígidos y parecidos a los tradicionales en papel, como el pdf, en lugar de otros más actuales, flexibles y con mayor potencial como xml, html, epub, etc., que posibilitan multimedia e interactividad.

Otras de esas resistencias al cambio tienen que ver con las inercias y tradiciones académicas procedentes de épocas en las que la competitividad investigadora era prácticamente nula y el rigor en los criterios de selección de los artículos casi una 
excentricidad de algunos editores. Así, analizando el caso de las revistas españolas de Ciencias Sociales vemos que, todavía, son apenas la mitad (un 56\%) las que aplican una revisión por pares, de las cuales apenas dos tercios (un 62\%) usan sistemáticamente el doble ciego (Giménez-Toledo, 2014).

Un último caso de esta resistencia al cambio procede de los enfoques antiguos que ven las revistas científicas más como una cuestión de imagen institucional, en las que mostrar qué se está haciendo en el Centro editor (Ruiz-Corbella, Galán \& Diestro, 2014) o, lo que es peor, dando una vía de salida a trabajos sin nivel suficiente para ser publicadas en revistas externas. Esta actitud resulta arcaica, priorizando una supuesta imagen institucional sobre la libre competencia de ideas, filtradas por expertos, propiciando el mantenimiento de muchas revistas cuya contribución real al conocimiento científico es, cuando menos, dudosa (Rodríguez-Yunta \& Giménez-Toledo, 2013).

\section{Oportunidades}

Los procesos institucionales de evaluación de la investigación que se desarrollaron fundamentalmente desde los años 90 (Giménez-Toledo, 2014, 2015; Ruiz Corbella, Galán \& Diestro, 2014) supusieron un acicate para que los editores mejorasen las publicaciones que gestionaban (para el caso de la investigación educativa, consúltese Fernández-Cano, 1995a, 1997).

El primer gran referente sobre los criterios de calidad fue, en 1995, el mexicano LATINDEX: establecieron unos criterios públicos, objetivos, claros y verificables, todo ello adaptado a la comunidad hispanohablante. Los editores podían verificar los criterios de calidad que aún le quedaban por cumplir, de modo que tenía una función clasificadora para las agencias evaluadoras, pero también una función pedagógica para los editores. A nuestro entender, el listado de criterios requeriría ya una ampliación, cuantitativa y cualitativa, ya que pasados unos años se ha producido un 'efecto techo'. La implantación de plataformas de gestión editorial informatizada (como OJS, por poner un ejemplo) hace que incluso una revista recién creada cumpla automáticamente con la mayoría de los criterios Latindex. También sirvió para fomentar el sentimiento de comunidad, unida por el idioma y la cultura. Igualmente contribuyó a estos efectos RedALyC, que centraliza publicaciones desde toda Iberoamérica (López, 2006). SciELO (Scientific Electronic Library Online) ha marcado otro hito en la proyección de las revistas Latinoamericanas (en España sólo da cobertura a revistas de Salud), con la creación de un Scielo Citation Index, integrado en Web of Science desde enero de 2014.

Desde España también se desarrollaron proyectos que impulsaron y desarrollaron la calidad editorial, a la vez que se convertían en medios de reconocimiento oficial. Destacamos proyectos de sistemas integrados de indicadores como DICE o RESH, índices de citas como In-RECS, In-RESH o In-RECJ o categorizaciones de revistas como CIRC o CARHUS (Giménez-Toledo, 2014) o evaluaciones rigurosas como las de FECYT. Debido a circunstancias ya comentadas, casi todos estos instrumentos han ido desapareciendo, pero las oportunidades que brindaron a toda una generación de editores para la mejora de la calidad de edición es un legado perdurable (Giménez-Toledo, 2014).

En apenas dos décadas ha habido un cambio radical en la difusión y acceso al conocimiento científico (Cooper, 2014). La aparición y desarrollo de revistas electró- 
nicas ha supuesto una gestión más económica, mayor difusión e incardinarse en una ciencia más global. A pesar de resistencias iniciales, el modelo de revista electrónica ha venido para quedarse definitivamente, convenciendo incluso a los más escépticos (Aliaga, 2014; Cabrera-Flores, Luna-Serrano \& Vidauri, 2014; Díaz Barriga, 2000; López, 2006). Algunos expertos incluso vaticinan ya la desaparición de las revistas impresas (Delgado López-Cózar, 2015).

Como ayuda a este proceso de creación (o conversión) de revistas electrónicas, se han desarrollado plataformas especializadas de gestión de contenidos, entre las que destaca por su amplia difusión un software de código abierto, el Open Journal System (OJS). La puesta en común de herramientas informáticas entre una gran cantidad de revistas científicas potencialmente permite cruzar información y crear índices de citas, indicadores de uso, sistemas de búsqueda compartida, etc., ofertando así la posibilidad de constituir 'paquetes de revistas' fácilmente gestionables por bibliotecas y utilizadas por lectores. Pueden constituirse a medio plazo en una alternativa muy económica a los 'paquetes de revistas' comerciales, reduciendo así los costosos sistemas de intermediación que apenas aportan valor añadido a la comunicación científica (Mañana \& Giménez-Toledo, 2015).

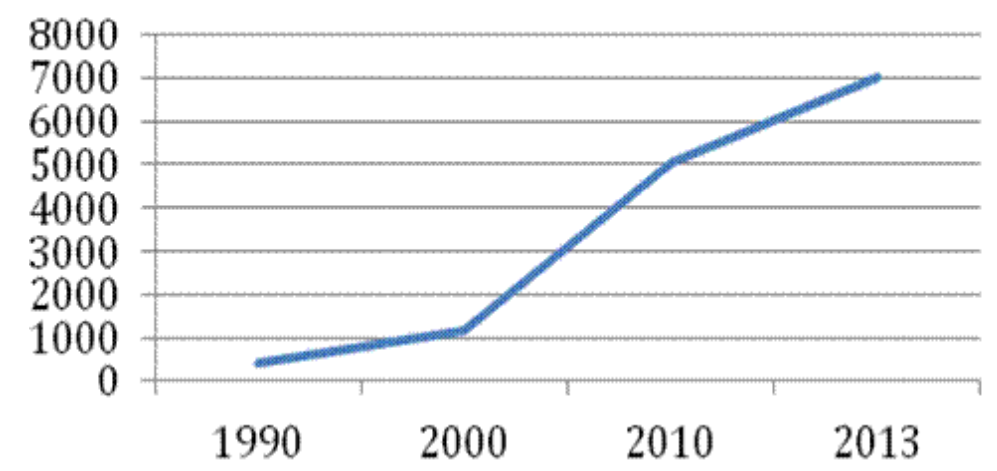

Figura 3. Incremento en el número de revistas que utilizan OJS Fuente: PKP (tomado de Ruiz-Corbella, Galán \& Diestro, 2014)

Otra de las potenciales oportunidades que ofrece la tecnología es la mejor identificación de la información. Su utilidad viene ejemplificada por la rápida implantación del DOI (Digital Object Identifier), que permite la preservación del contenido electrónico y la reducción de errores de identificación de los documentos. También se está aplicando a la identificación de los autores mediante sistemas como el código ORCID, potente alternativa Abierta al ResearchID de Clarivate o al Scopus Author Identifier de Elsevier (Fenner, 2011).

El desarrollo de las redes sociales (incluidas las académicas) propicia nuevas oportunidades para difundir mejor la producción científica (Cooper, 2014; Ruiz-Corbella, Galán \& Diestro, 2014). Estas herramientas no son una amenaza a las revistas, sino un complemento, pues aunque su capacidad de contenido es mucho menor, su velocidad y 
público potencial no tienen comparación. Torres-Salinas, Cabezas-Clavijo y Jiménez (2013) encontraron una notable correlación entre tuits y descargas de artículos, pero no tanto entre tuits y número de citas recibidas. Ello nos muestra que esas estrategias de difusión nos pueden proporcionar más lectores pero no, necesariamente, mayor impacto académico.

El desarrollo de iniciativas de evaluación (FECYT, etc.), de difusión (Redalyc, Redib, etc.) y la utilización de plataformas informáticas (OJS, etc.) han fomentado, de manera indirecta, el desarrollo entre los editores de una cierta conciencia de comunidad, con problemas y preocupaciones semejantes, que han permitido romper el aislamiento que tradicionalmente ha caracterizado la labor editorial en un ámbito tan atomizado como el educativo. Se abre un mundo de posibilidades sobre iniciativas conjuntas (actuar como grupo de presión ante autoridades y agencias evaluadoras, procesos de formación, de resolución de dudas, de denuncia de actividades irregulares...).

Desde sus inicios, el movimiento de Acceso Abierto (AA) mostró una vitalidad notable, tanta que hizo incluso tambalearse los principios comerciales de las grandes corporaciones editoriales. La idea de poner el conocimiento científico al alcance de todos tenía un evidente atractivo, tanto por la mayor difusión del conocimiento como por justicia social (Giménez-Toledo, 2014). En 2011 España promulgó su Ley de la Ciencia y la Innovación de España con un artículo específico sobre AA que pretende institucionalizar el apoyo a ese modelo (Melero, 2014). Argentina, Perú y México aprobaron en 2012 también leyes en el mismo sentido.

No obstante, algo habría que comentar de la sutil perversión que, so capa de Open Access, se está generando últimamente, al tener que pagar los autores altos precios a la revista editora. Pagar por publicar se está convirtiendo en una práctica usual, cara y excluyente.

\section{Amenazas}

Las revistas científicas son un fenómeno dinámico. Como tal, también son diversas las amenazas que se plantean a su desarrollo. Una de estas amenazas externas tiene que ver con la intensificación de los procesos de evaluación, debido a sus efectos secundarios. La cantidad de investigadores (en ejercicio o en formación) que desean publicar en las revistas mejor valoradas (para conseguir su tesis doctoral por compendio de publicaciones, para mejorar su curriculum, para obtener mejores condiciones laborales, etc.) es muchísimo mayor que el número de artículos que estas revistas pueden publicar. El resultado es el embudo, una enorme (y creciente) presión que se concentra sobre unas pocas revistas (Fonseca-Mora \& Aguaded, 2014), con el resultado de un aumento espectacular de la carga de trabajo sobre el equipo editorial, un aumento de las tasas de rechazo (y las consiguientes frustraciones), una reiteración de envíos a revistas progresivamente menos exigentes (lo que sobrecarga la parte alta de la pirámide) y una ausencia de envíos a revistas que no han visto aún reconocida oficialmente su calidad, ya que no aportan incentivos a los autores, lo que provoca una mayor mortalidad de revistas.

Otra amenaza externa tiene que ver con la metodología utilizada por los evaluadores de la investigación. Para simplificar su ingente tarea, los gestores que establecen las normas para estas evaluaciones suelen buscar medidas simples de productividad o "calidad" (Ishikawa, 2014), ya que un valor único (como, por ejemplo, el factor de 
impacto -FI), objetivo, rápido y verificable, aporta fiabilidad al proceso, a la vez que lo simplifica, permitiendo hacer rankings, establecer puntos de corte, etc. Sin embargo, conviene tener presente que asignar números a la realidad es medir, que no es lo mismo que evaluar, que implica un proceso de juicio sistemático, con establecimiento de criterios y con valoración de componentes subjetivos bien informados. Por eso hay autores, como Fernández-Ríos y Rodríguez-Díaz (2014, p. 156) que señalan que "el índice de impacto es un generador de pensamiento irreflexivo". Algo de eso ha debido detectarse cuando sistemas de evaluación que han sido pioneros, como el británico, han establecido en la normativa que guía la investigación y su evaluación (el Research Excellence Framework) de manera expresa excluye de los procesos evaluativos el uso de indicadores bibliométricos cuantitativos, el de datos sobre citas o la categorización de revistas (Giménez-Toledo, 2015). La reciente reforma de los procesos de acreditación del profesorado en España va en la misma dirección, aumentando el carácter cualitativo de las evaluaciones. Si ésta es la dirección correcta o tan sólo una amenaza añadida al proceso (por pérdida de referentes objetivos), sólo el tiempo lo dirá.

Una de las potenciales amenazas puede venir de la importación de modelos comunicativos foráneos. Es cierto que el inglés se ha convertido en Ciencias Naturales en lingua franca de comunicación casi universal. Hay fuertes presiones (algunas razonables) para que también lo sea en el ámbito de las Ciencias Sociales. Sabemos que buena parte de la comunicación en las disciplinas más sociales, más culturales, se desarrolla en el idioma vernáculo (Chou, 2014; Ishikawa, 2014). Ello tiene sentido porque ese tipo de conocimiento está enraizado en la cultura de origen $y$, con frecuencia, va dirigido a esa misma sociedad. Expresarlo en un idioma diferente podría facilitar un mayor impacto académico, pero dificultaría su impacto social, al quedar fuera del alcance de la mayoría de los integrantes de esa sociedad. Países tecnológicamente tan avanzados como Japón o Taiwan no tienen reparos en defender que la expresión de buena parte de su investigación social se haga en su idioma vernáculo (Chou, 2014; Ishikawa, 2014).

Algunos autores (Fonseca-Mora \& Aguaded, 2014) defienden que la no utilización del inglés en la comunicación científica supone un "pobreza lingüística que significa el rechazo de la comunidad científica internacional" (p. 7). Desde un planteamiento inverso, Post (2014) señala que la presión a favor del inglés supone una pérdida de otras perspectivas (homogeneización) y de buena parte de la literatura, particularmente en Ciencias Sociales, fundamentalmente para los angloparlantes monolingües. Sigue siendo una cuestión no resuelta.

No hemos de olvidar que los sistemas nacionales de evaluación, en general, priorizan el uso de los índices dominados por las dos grandes empresas comerciales que copan ese mercado: Clarivate y Elsevier, con una perspectiva global y, generalmente, anglófila, aunque cada vez de una manera más matizada. Esas bases de datos internacionales no evalúan adecuadamente los campos de Humanidades y Ciencias Sociales (GiménezToledo, 2015). Por eso se hace conveniente complementar aquella información, por lo demás también útil, con otros indicadores que consideren de manera más amplia disciplinas más vinculadas a lo social y cultural, por lo que es una auténtica desgracia para las Ciencias Sociales el hecho de que hayan desaparecido tantos instrumentos de ese tipo: DICE, RESH, In-RECS, In-RECJ, In-RESH, ERIH (Aliaga, 2014; Delgado LópezCózar, 2015; Giménez-Toledo, 2014, 2015), que demostraron ampliamente su utilidad. 
Aunque lo hemos apuntado brevemente más arriba, no queremos dejar de subrayar un aspecto singular. Se trata de la contraposición entre impacto académico (medido generalmente en número de citas que recibe un artículo) e impacto social, que podríamos definir como el beneficio o progreso real que consigue un estudio sobre las prácticas educativas, por especificar en nuestro campo (Alcántara \& Márquez Jiménez, 2017; Fonseca-Mora \& Aguaded, 2014). En las evaluaciones de los investigadores se utiliza casi exclusivamente el impacto académico, que puede medirse más fácilmente. Sin embargo, orientar a los jóvenes investigadores hacia ese tipo de impacto, y no hacia el que provoca un auténtico progreso social, es hacer un flaco servicio a la comunidad a la que deben servir.

\section{Conclusiones}

Hemos hecho una revisión de los principales factores que afectan a la edición de revistas científicas siguiendo la estructura DAFO. Sin duda, no todos están cubiertos, dadas las limitaciones de espacio de un artículo. No pretendemos cerrar ninguno de los temas, sino por el contrario, mostrarlos a la comunidad académica, que es quien debe debatir sobre ellos.

El ámbito de la "journalología" iberoamericana se revela como un campo proceloso y complejo, con múltiple problemas pero también con grandes posibilidades, dado sobre todo el ingente potencial de investigadores educativos que comparten las dos lenguas ibérica (español y portugués), habladas por más de 600 millones de personas.

Múltiples cuestiones quedan pendientes; por ejemplo, crear una gran base de datos con revistas no solo españolas sino incorporando también las iberoamericanas, ampliando y fortaleciendo entonces la actual base ISOC-CINDOC.

La enorme mejora de las publicaciones científicas de educación en las dos últimas décadas (calidad, difusión, etc.) no debe hacernos olvidar que quedan pendientes aspectos fundamentales, como la evaluación de sus aspectos más sociales (impactos locales, idioma, cambios propiciados en la comunidad, etc.), los más vinculados al entorno en el que se producen y al que deben servir.

\section{Referencias}

Alcántara, A. \& Márquez A. (2017). La medida de la investigación en educación y su impacto social: las revistas de educación de Iberoamérica en los índices bibliométricos internacionales. Revista de la Asociación de Sociología de la Educación (RASE), 10(2), 225-239. doi: http://doi.org/10.7203/RASE.10.2.10087

Aliaga, F. M.; Almerich, G. \& Suárez-Rodríguez, J. (2013). El European Reference Index for the Humanities (ERIH) como criterio de calidad de las revistas académicas: análisis de la lista revisada de educación. Revista Española de Documentación Científica, 36(2):en008. doi: http://doi.org/10.3989/redc.2013.2.901

Aliaga, F. M. (2014). Veinte años de publicación electrónica y de acceso abierto: la madurez de una pionera. RELIEVE, 20(1), art. 0. doi: http://doi.org/10.7203/ relieve.20.1.3856 
Cabrera-Flores, M.; Luna-Serrano, E. \& Vidauri, G. (2014). Las revistas mexicanas de investigación educativa rumbo a la corriente principal de difusión de la ciencia. RELIEVE, 20(2), art. M5. doi: http://doi.org/10.7203/relieve.20.2.4405

Chang, H. H. \& Huang, W. C. (2006). Application of a quantification SWOT analytical method. Mathematical and Computer Modelling, 43(1), 158-169.

Chou, C. P. (2014). The SSCI Syndrome in Taiwan's Academia. Education Policy Analysis Archives, 22(29). doi: http://doi.org/10.14507/epaa.v22n29.2014.

Cooper, A. (2014). The use of online strategies and social media for research dissemination in education. Education Policy Analysis Archives (EPAA), 22(88), 2-27. doi: http://doi. org/10.14507/epaa.v22n88.2014

Delgado López-Cózar, E. (2015). Las revistas electrónicas en acceso abierto: pasado, presente y futuro. RELIEVE, 21(1), art. M1. doi: http://doi.org/10.7203/relieve.21.1.5005

Delgado Troncoso, J. E.; Martínez-Hernández, D.; López, B. L.; Manco-Vega, A.; Aliaga, F. M.; Tejada, M. A. \& Romero, C. (2014). Acceso, uso y publicación en revistas científicas entre los investigadores en Ciencias Sociales de Latinoamérica. doi: http://doi.org/10.6084/ m9.figshare.1041561

Delgado, J. E. (2011). Journal publication in Chile, Colombia and Venezuela: University Responses to global, regional and national pressures and trends. Doctoral dissertation. University of Pittsburgh. Recuperado de http://dscholarship.pitt.edu/9049/

Delgado, J.E. (2014). Scientific Journals of Universities of Chile, Colombia, and Venezuela: Actors and roles. Education Policy Analysis Archives, 22(34). doi: http:// doi.org/10.14507/epaa.v22n34.2014

Diestro, A, Ruiz Corbella, M., \& Galán, A. (2017). Calidad editorial y científica en las revistas de educación. Tendencias y oportunidades en el contexto 2.0. Revista de Investigación Educativa, 35(1), 235-250. doi: http://doi.org/10.6018/rie.35.1.244761

Díaz Barriga, A. (2000). Pasado y presente de las revistas de educación en México. Perfiles Educativos, 22(88), 2-5.

Dorta-González, P. \& Dorta-González, M.I. (2013). Hábitos de publicación y citación según campos científicos: principales diferencias a partir de las revistas JCR. Revista Española de Documentación Científica, 36(4). doi: http://doi.org/10.3989/redc.2013.4.1003

Fenner, M. (2011). Author identifier overview. Recuperado de http://edoc.hu-berlin.de/ libreas/18/fenner-martin-18/PDF/fenner.pdf

Fernández-Cano, A. (1995a). Métodos para evaluar la investigación en psicopedagogía. Madrid: Síntesis.

Fernández-Cano, A. (1995b). La evaluación de la investigación educativa. Revista Española de Pedagogía, 52(200), 131-146.

Fernández-Cano, A. (1997). Evaluación de la investigación educativa española: Una revisión integrativa de realizaciones en 25 años. Revista Española de Pedagogía, 55(207), 277-301.

Fernández-Cano, A. \& Bueno-Sánchez, A. (2002). Multivariate evaluation of Spanish educational research journals. Scientometrics, 55(1), 87-102. doi: http://doi.org/10.1023/ B:SCIE.0000045112.11562.117

Fernández-Ríos, L. \& Rodríguez-Díaz, F. J. (2014). The "impact factor style of thinking": A new theoretical framework. International Journal of Clinical and Health Psychology, 14(2), 154-160. 
Fischman, G. E.; Alperín, J. P., \& Willinsky, J. (2010). Visibility and quality in Spanishlanguage Latin American scholarly publishing. Information Technologies $\mathcal{E}$ International Development, 6(4), 1-21.

Fonseca-Mora, M.C. \& Aguaded, I. (2014). Las revistas científicas como plataformas para publicar la investigación de excelencia en educación: estrategias para atracción de investigadores. RELIEVE, 20(2), art. M3. doi: http://doi.org/10.7203/relieve.20.2.4274

Giménez-Toledo, E. (2014). Imposturas en el ecosistema de la publicación científica. Revista de Investigación Educativa, 32(1), 13-23. doi: http://doi.org/10.6018/rie.32.1.190251

Giménez-Toledo, E. (2015). La evaluación de la producción científica: breve análisis crítico. RELIEVE, 21(1), art. M2. doi: http://doi.org/10.7203/relieve.21.1.5160

Hill, T. \& Westbrook, R. (1997). SWOT analysis: it's time for a product recall. Long Range Planning, 30(1), 46-52.

Ishikawa, M. (2014). Ranking regime and the future of vernacular scholarship. Education Policy Analysis Archives, 22(30). doi: http://doi.org/10.14507/epaa.v22n30.2014. This article is part of EPAA/AAPE's Special Issue on The Future of Education Research Journals, Guest Edited by Dr. David Post.

Kling, R. \& Callahan, E. (2003). Electronic journals, the Internet and scholarly communication. Annual Review of Information Science and Technology, 37, 127-177.

Lee, K. L. \& Lin, S. C. (2008). A fuzzy quantified SWOT procedure for environmental evaluation of an international distribution center. Information Sciences, 178(2), 531-549.

Mañana, J. \& Giménez-Toledo, E. (2015). Revistas de educación e investigación educativa. modelos de negocio y desempeño bibliométrico. Revista de Educación, 369, 31-58. doi: http://doi.org/10.4438/1988-592X-RE-2015-369-289.

Martínez-Rizo, F. (1999). La Revista de la Educación Superior en el panorama editorial mexicano. Revista de la Educación Superior, 27(111), 101-110.

Melero, R. (2014). RELIEVE: Veinte años inmersos en la cronología del acceso abierto a la ciencia. RELIEVE, 20(2), art. M2. doi: http://doi.org/10.7203/relieve.20.2.4300

Merton, R. K. (1973). The sociology of science: Theoretical and empirical investigations. University of Chicago Press. [Traducción española de 1977: La sociología de la ciencia. Madrid: Alianza].

Post, D. (2014). The future of education research publishing: Challenges and responses. Education Policy Analysis Archives, 22(26), 1-9. doi: http://doi.org/10.14507/epaa. v22n26.2014

Purnell, P. J. \& Quevedo-Blasco, R. (2013). Benefits to the Spanish research community of regional content expansion in Web of Science. International Journal of Clinical and Health Psychology, 13(2), 147-154.

Rodríguez-Yunta, L. \& Giménez-Toledo, E. (2013). Fusión, coedición o reestructuración de revistas científicas en humanidades y ciencias sociales. El Profesional de la Información, 22(1), 36-45. doi: http://doi.org/10.3145/epi.2013.ene.05

Ruiz-Corbella, M.; Galán, A. \& Diestro, A. (2014). Las revistas científicas de Educación en España: evolución y perspectivas de futuro. RELIEVE, 20(2), art. M1. doi: http:// doi.org/10.7203/relieve.20.2.4361

Seligman, M. E. P. (1975). Helplessness: On depression, development, and death. San Francisco: W. H. Freeman

Testa, J. (2011). The globalization of Web of Science: 2005-2010. Nueva York: Thomson Reuters. 
Torres-Salinas, D., Cabezas-Clavijo, Á. \& Jiménez, E. (2013). Altmetrics: Nuevos indicadores para la comunicación científica en la Web 2.0. Comunicar, 41(22), 53-60. doi: http://doi.org/10.3916/c41-2013-05

Weihrich, H. (1982). The TOWS matrix: A tool for situational analysis. Long Range Planning, 15(2), 54-66.

Wang, X. P., Zhang, J., \& Yang, T. (2014). Hybrid SWOT approach for strategic planning and formulation in China worldwide express mail service. Journal of Applied Research and Technology, 12(2), 230-238. doi: https://doi.org/10.1016/S1665-6423(14)72339-9

Fecha de recepción: 28 de noviembre de 2017

Fecha de revisión: 30 de noviembre de 2017

Fecha de aceptación: 23 de abril de 2018 
